\title{
Nanocomposite Ag Thin Films With Interactive Topography
}

\author{
A. P. Piedade* ${ }^{*}$ J. Nunes* and M.T.Vieira*
}

* CEMUC - Departamento de Engenharia Mecânica, Universidade de Coimbra, Rua Luís Reis Santos, 3030-788 Coimbra, Portugal

ana.piedade@dem.uc.pt

Synthesis of nanostructures has triggered continuous interest in the nanoscience field due to their potential application such as catalysis and biomaterials[1]. Among these systems the possibility of producing nano- microporous surfaces with various controlled structures and pore sizes has increased the interest in the field owing to a better control over the particle's size and location[2].

In these study thin films of ceramic material (alumina and silica) containing nanoclusters of silver have been deposited by rf magnetron co-sputtering. The thin films were characterised asdeposited and the mean size of the clusters was estimated by transmission electron microscopy (TEM). The silver was removed from the samples by chemical etching and immersion in simulated body fluids. The morphology of the as-deposited and silver depleted thin films was observed by scanning electron microscopy (SEM). The change in surface roughness and porosity was evaluated by treating the images with ImageJ program[3] This procedure gives a new path for the development of nano- microporous thin films with "smart" topography. In fact, the use of elemental silver it is expected to provide unassisted sustained release of metal ions at antimicrobial concentrations when in contact with aqueous electrolyte.

The results showed that depending on the silver content of each thin film the obtained cluster varied from micro to nano $(<100 \mathrm{~nm}$ ) (Fig 1). Thus for further characterization only the thin films with silver atomic percentages lower the $20 \%$ were used. The TEM characterisation also demonstrated that the structure of the films, independently of the target materials being alumina or silica, consist of an amorphous ceramic matrix where the silver crystalline nanoclusters are "embebed". The release of silver produced a significant alteration of the surface morphology (Fig.2 and 3), which is more important with the increase of silver removal

This strategy is important when considering that the interactive nature of the thin films allows the production of micro and nanoporosity, which has been related with increase of cell adhesion and proliferation when compared with the smooth surface of the same chemical composition[4]. These results indicate that the deposited thin films, according to the strategy of interactive surface, are promising for long-term applications in implanted materials, where an adaptative interface with the biological tissue tends to mimic the natural tissues.

References:

[1] Y.Chen, H.Kim, Mater. Lett., 61 (2007) 5040

[2] L.Armelao, G. Bottaro, R.Campostrini, S.Gialanella, M.Ischia, F.Poli, E.Tondello, Nanotechnology, 18 (2007) 155606.

[3] W.Rasband, National Institutes of Health, USA http://rsb.info.nih.gov/ij/ - ImageJ is in the public domain

[4] SD. Alvareza et al Biomaterials, 30 (2009) 26. 

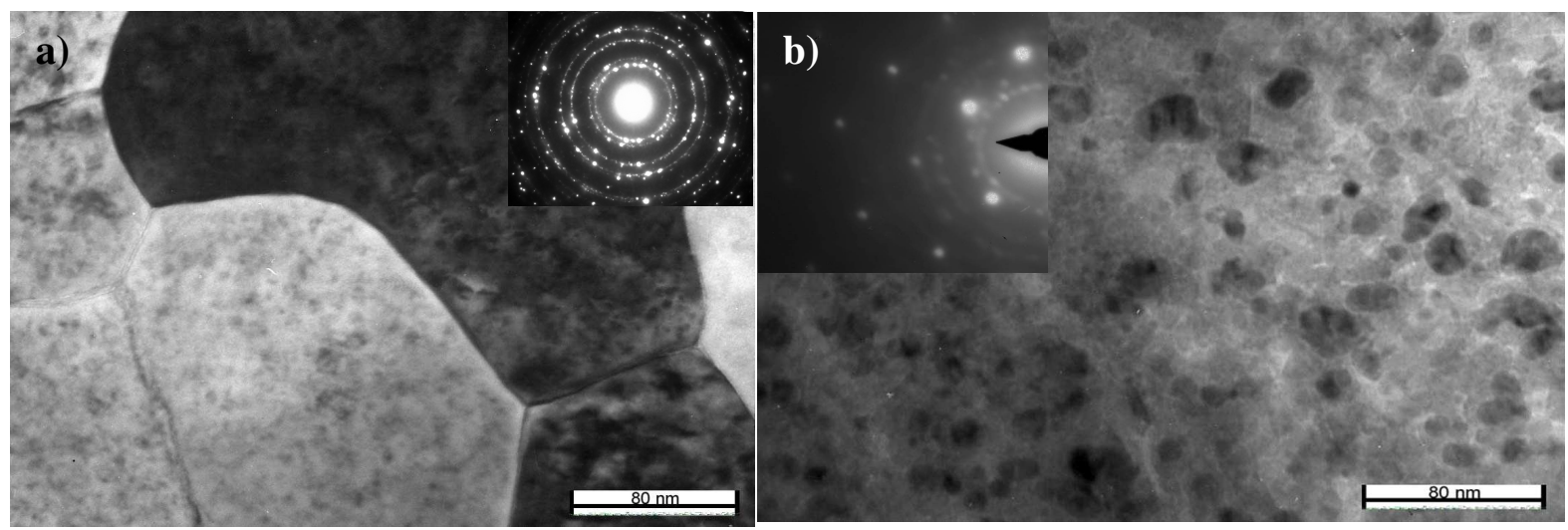

Fig. 1. Transmission electron micrographs of (a) high and (b) intermediate silvent content on $\mathrm{Al}_{2} \mathrm{O}_{3} / \mathrm{Ag}$ composites thin films. Inserts of the electron diffration patterns show the crystalline structure of silver and the amorphous nature of the ceramic matrix.
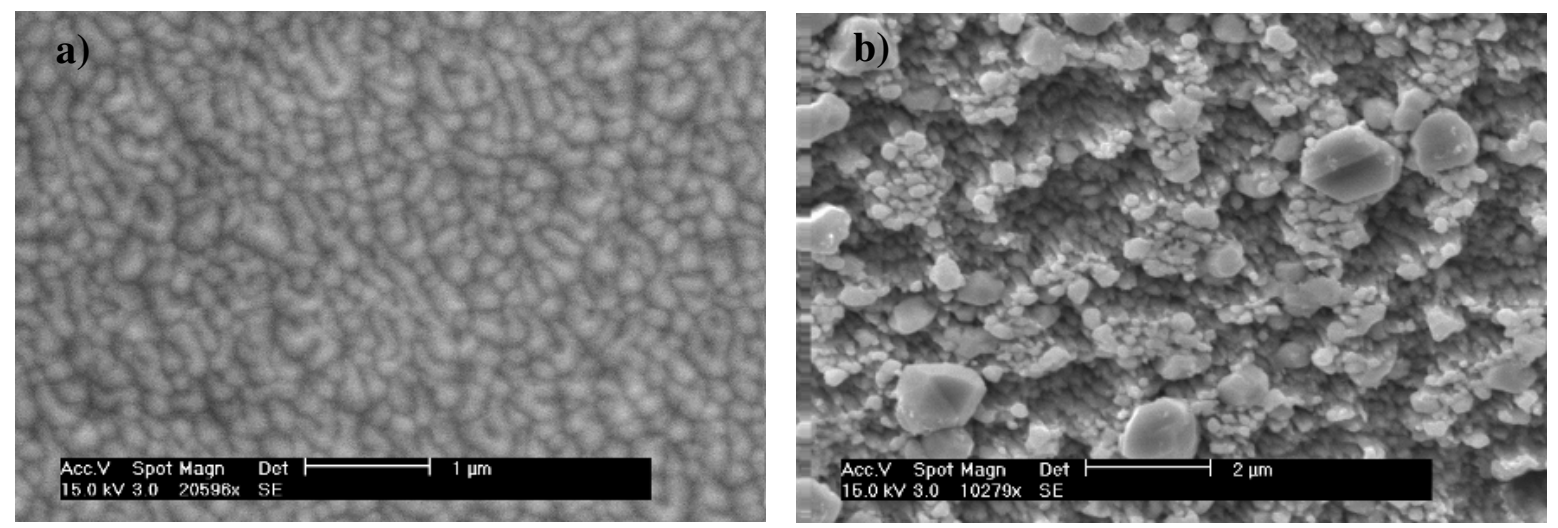

Fig. 2. Scanning electron micrographs of the surface of $\mathrm{ZrO} 2 / \mathrm{Ag}$ nanocomposite thin film with 30 at $\%$ of silver. Surface morphology of the as-deposited thin film (a) and after silver removal (b).
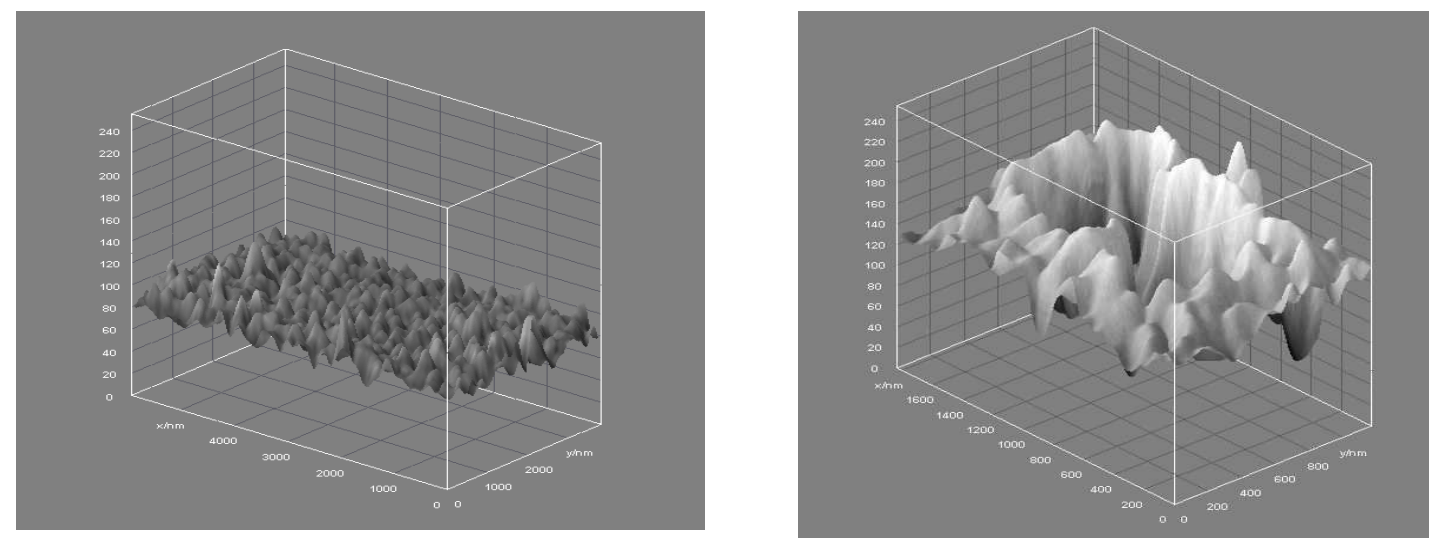

Fig.3 - 3D-surface plot images of the morphologies of Fig. 2 after Image J treatement. The 3D images enphasise the increase of surface porosity. 